\title{
ON A MINIMAL NORMAL DILATION PROBLEM
}

\author{
AMEER ATHAVALE
}

(Communicated by Palle E. T. Jorgensen)

\begin{abstract}
By combining the theory of disintegration of measures with certain approximation theorems in several complex variables, we derive some positive results concerning a minimal normal dilation problem for operator tuples whose coordinates are special functions of subnormal operators as well as their adjoints.
\end{abstract}

\section{Preliminaries}

All the Hilbert spaces occurring in this paper are complex and separable. For a Hilbert space $\mathscr{H}, \mathscr{B}(\mathscr{H})$ denotes the set of bounded linear operators on $\mathscr{H}$. Any operator tuple discussed is assumed to consist of commuting bounded linear operators, though this may not always be stated explicitly. Let $S=\left(S_{1}, \ldots, S_{m}\right)$ and $T=\left(T_{1}, \ldots, T_{m}\right)$ be two operator tuples such that $S_{i} \in \mathscr{B}(\mathscr{H})$ and $T_{i} \in \mathscr{B}(\mathscr{K})$ for all $i$, where $\mathscr{K}$ is a Hilbert space containing $\mathscr{H}$ as a closed subspace. We say that $S$ dilates to $T$ if $S_{1}^{\alpha_{1}} S_{2}^{\alpha_{2}} \cdots S_{m}^{\alpha_{m}}=P(\mathscr{K}, \mathscr{H}) T_{1}^{\alpha_{1}} T_{2}^{\alpha_{2}} \cdots T_{m}^{\alpha_{m}} / \mathscr{H}$ for all nonnegative integers $\alpha_{i}$, where $P(\mathscr{K}, \mathscr{H})$ denotes the orthogonal projection of $\mathscr{K}$ onto $\mathscr{H}$. If, in addition, $\mathscr{H}$ is invariant for each $T_{i}$, then $S$ is said to extend to $T$. If $S$ dilates (extends) to $T$ and each $T_{i}$ is normal, then $T$ will be referred to as a normal dilation (extension) of $S$. Also, $T$ will be called a minimal normal dilation of $S$ if $T$ is a normal dilation of $S$ and $\mathscr{K}$ does not have a proper closed subspace containing $\mathscr{H}$ and reducing each $T_{i}$. An operator tuple $S$ admitting a normal extension is said to be subnormal, and among all the normal extensions of $S$ there is a 'minimal normal extension', which is unique up to unitary equivalence [I].

Suppose $N$ is a normal extension of a subnormal tuple $S$. Using the spectral theory for commuting normals, one may consider $\varphi(N) \equiv\left(\varphi_{1}(N), \ldots, \varphi_{k}(N)\right)$, where $\varphi=\left(\varphi_{1}, \ldots, \varphi_{k}\right)$ is a tuple of bounded Borel functions on the joint spectrum of $N$. In certain special cases to be discussed, $\varphi(N)$ turns out to be a normal dilation of

$$
P(\mathscr{K}, \mathscr{H}) \varphi(N) / \mathscr{H} \equiv\left(P(\mathscr{K}, \mathscr{H}) \varphi_{1}(N) / \mathscr{H}, \ldots, P(\mathscr{K}, \mathscr{H}) \varphi_{k}(N) / \mathscr{H}\right)
$$

Received by the editors October 18, 1992.

1991 Mathematics Subject Classification. Primary 47A20, 47B20.

Key words and phrases. Subnormal, (minimal) normal extension, (minimal) normal dilation. 
In such a case, we raise the following question: Is $\varphi(N)$ a minimal normal dilation of $P(\mathscr{K}, \mathscr{H}) \varphi(N) / \mathscr{H}$ ? We will provide an answer to this question using the theory of disintegration of measures. Specific applications follow by invoking some highly nontrivial approximation theorems in several complex variables. The arguments in this paper are entirely motivated by those in [C2], and a knowledge of the first three sections of [C2] is more or less essential for an understanding of this paper.

If $S=\left(S_{1}, \ldots, S_{m}\right)$ is a subnormal tuple with a normal extension $N=$ $\left(N_{1}, \ldots, N_{m}\right) \quad\left(S_{i} \in \mathscr{B}(\mathscr{H}), N_{i} \in \mathscr{B}(\mathscr{K})\right)$, then

$$
\begin{aligned}
& S_{1}^{*_{1}^{p}} S_{2}^{*_{2}^{p}} \cdots S_{m}^{*_{m}^{p}} S_{1}^{q_{1}} S_{2}^{q_{2}} \cdots S_{m}^{q_{m}} \\
& \quad=P(\mathscr{K}, \mathscr{H}) N_{1}^{*_{1}^{p}} N_{2}^{*_{2}^{p}} \cdots N_{m}^{*_{m}^{p}} N_{1}^{q_{1}} N_{2}^{q_{2}} \cdots N_{m}^{q_{m}} / \mathscr{H}
\end{aligned}
$$

for all nonnegative integers $p_{i}$ and $q_{i}$. Suppose $I \cup J$, with $I=\left\{i_{1}, \ldots, i_{s}\right\}$ and $J=\left\{j_{1}, \ldots, j_{t}\right\}$, is a partition of $\{1,2, \ldots, m\}$. By $P(S ; I, J)$ we will indicate a polynomial of the type

$$
\sum a_{p_{1} p_{2} \cdots p_{s} q_{1} q_{2} \cdots q_{t}} S_{i_{1}}^{*_{1}^{p}} S_{i_{2}}^{*_{2}^{p}} \cdots S_{i_{s}}^{*_{s}^{p}} S_{j_{1}}^{q_{1}} S_{j_{2}}^{q_{2}} \cdots S_{j_{i}}^{q_{i}}
$$

Recall that an operator tuple $S=\left(S_{1}, \ldots, S_{m}\right)$ is said to be doubly commuting if $S_{i}^{*} S_{j}=S_{j} S_{i}^{*}$ (and, of course, $S_{i} S_{j}=S_{j} S_{i}$ ) for $i \neq j$. The multiplication tuples on the tensor products of functional Hilbert spaces, such as the Hardy space or the Bergman space of the unit polydisk, are typical examples of doubly commuting subnormal tuples. It is well known that if $S_{1}, \ldots, S_{m}$ are doubly commuting subnormals on a Hilbert space, then $S=\left(S_{1}, \ldots, S_{m}\right)$ is (jointly) subnormal [I]. If $S$ is a tuple of doubly commuting subnormals with a normal extension $N$, then it is easy to see that, for any partition $I \cup J$ of $\{1, \ldots, m\}$ and any positive integer $k,\left(P_{1}(N ; I, J), \ldots, P_{k}(N ; I, J)\right)$ is a normal dilation of $\left(P_{1}(S ; I, J), \ldots, P_{k}(S ; I, J)\right)$, where each $P_{i}$ indicates a polynomial of the type mentioned previously. (For example, with $m=3, k=1$, $I=\{1,3\}$, and $J=\{2\}, N_{3}^{*} N_{2}+N_{1}^{*^{2}}$ is a normal dilation of $S_{3}^{*} S_{2}+S_{1}^{* 2}$.) The appearance of the adjoints of subnormal operators in the above expressions necessitates an extension of some of the theory developed in [C2], which is the topic of the next section. While the applications in $\S 3$ are somewhat specialized, the results in $\S 2$ are stated in such a way as to allow for the possibility of more general applications in the future.

\section{Disintegration OF MEASURES}

We begin by quoting the following theorem from [C2].

Theorem 1. If $N=\left(N_{1}, \ldots, N_{m}\right)$ is a commuting tuple of normal operators on a Hilbert space $\mathscr{K}$, then there is a compact metric space $X$, bounded Borel functions $\chi_{1}, \ldots, \chi_{m}$ on $X$, and a regular Borel measure $\mu$ on $X$ such that each $N_{j}$ is unitarily equivalent to multiplication by $\chi_{j}$ on $L^{2}(\mu)$.

Using the notation of Theorem 1 , if $S$ is a subnormal tuple with a normal extension $N$, then each $S_{j}$ is defined by multiplication by $\chi_{j}$ on some closed sub-

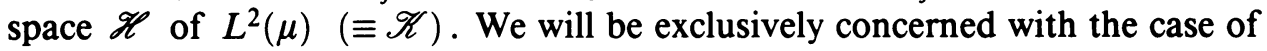
$\chi_{j}$ being the coordinate functions $z_{j}$. Thus, if $\varphi_{1}, \ldots, \varphi_{k}$ are bounded Borel functions on $X$ and $\varphi(N)$ is a normal dilation of $\varphi(S) \equiv P(\mathscr{K}, \mathscr{H}) \varphi(N) / \mathscr{H}$, 
then $\varphi(N)$ is a minimal normal dilation of $\varphi(S)$ if and only if

$$
\begin{aligned}
& L^{2}(\mu)=V\left\{\bar{\varphi}_{1}^{q_{1}} \bar{\varphi}_{1}^{q_{2}} \cdots \bar{\varphi}_{k}^{q_{k}} \varphi_{1}^{p_{1}} \varphi_{2}^{p_{2}} \cdots \varphi_{k}^{p_{k}} h:\right. \\
&\left.h \in \mathscr{H} ; p_{i}, q_{i} \text { are nonnegative integers }\right\}
\end{aligned}
$$

where $V$ denotes the closed linear span in the norm of $L^{2}(\mu)$. Note that if the space $\mathscr{H}$ is invariant for each $\varphi_{i}(N)$, then (1) is equivalent to requiring $\varphi(N)$ to be a minimal normal extension of $\varphi(S) \equiv \varphi(N) \mid \mathscr{H}$. (In case the functions $\varphi_{i}$ involve the conjugates $\bar{z}_{j}$, the requirement that $\mathscr{H}$ be invariant for each $\varphi_{i}(N)$ is rather stringent.) Checking the validity of (1) in specific cases involves two steps, namely:

(1) invoking an appropriate approximation theorem in several complex variables (involving the conjugate functions $\bar{z}_{j}$ ) and

(2) an appeal to Theorem 3 (or Corollary 1).

The following theorem on disintegration of measures was used crucially in [C2] and is restated here for ready reference.

Theorem 2 [B]. Suppose $X$ and $Z$ are compact metric spaces and $\mu$ is a positive regular Borel measure on $X$. If $\varphi: X \rightarrow Z$ is a Borel map, then there exists a unique mapping $\xi \rightarrow \lambda_{\xi}$ of $Z$ into the set $M(X)$ of regular positive Borel measures on $X$ satisfying

(a) $\lambda_{\xi}$ is a probability measure supported on $\varphi^{-1}(\xi)$ for all $\xi$ in $\varphi(X)$.

(b) For any continuous function $f$ on $X, \xi \rightarrow \int_{X} f d \lambda_{\xi}$ is a $\left(\mu \circ \varphi^{-1}\right)$ integrable function on $Z$.

(c) For any continuous function $f$ on $X$

$$
\int f d \mu=\int\left[\int f d \lambda_{\xi}\right] d\left(\mu \circ \varphi^{-1}\right)(\xi) .
$$

It is important to note the following particular consequence of Theorem 2:

$$
\int|g|^{2} d \mu=\int\left[\int_{\varphi^{-1}(\xi)}|g|^{2} d \lambda_{\xi}\right] d\left(\mu \circ \varphi^{-1}\right)(\xi) \text { for any } g \text { in } L^{2}(\mu) .
$$

The following theorem is an extension of [C2, Theorem 3.5].

Theorem 3. Suppose $S$ is a subnormal tuple of operators on a Hilbert space $\mathscr{H}$ with a normal extension $N$ of operators on $\mathscr{K}$ and $\varphi=\left(\varphi_{1}, \ldots, \varphi_{k}\right)$ is a $k$-tuple of bounded Borel functions on the joint spectrum of $N$ with $\varphi(N)$ being a normal dilation of $\varphi(S) \equiv P(\mathscr{K}, \mathscr{H}) \varphi(N) \mid \mathscr{H}$. With $\mu$ as in Theorem 1, let $\xi \rightarrow \lambda_{\xi}$ be the disintegration of $\mu$ with respect to $\varphi$ as described in Theorem 2; and let $\mathscr{K}_{\xi}=L^{2}\left(\lambda_{\xi}\right)$-closure of $\left\{h / \varphi^{-1}(\xi): h \in \mathscr{H}_{0}\right\}$, where $\mathscr{H}_{0}$ is some dense subset of $\mathscr{Z}$. Then $\varphi(N)$ is a minimal normal dilation of $\varphi(S)$ if and only if

$$
L^{2}(\mu)=\left\{f \in L^{2}(\mu): f / \varphi^{-1}(\xi) \in \mathscr{K}_{\xi} \text { a.e. }\left[\mu \circ \varphi^{-1}\right]\right\} .
$$

Proof. In view of (1), it suffices to show that $\mathscr{K}^{\prime} \equiv\left\{f \in L^{2}(\mu): f / \varphi^{-1}(\xi) \in \mathscr{K}_{\xi}\right.$ a.e. $\left.\left[\mu \circ \varphi^{-1}\right]\right\}$ equals

$$
\begin{aligned}
\mathscr{K}^{\prime \prime} \equiv V\left\{\bar{\varphi}_{1}^{q_{1}} \bar{\varphi}_{2}^{q_{2}} \cdots \bar{\varphi}_{k}^{q_{k}} \varphi_{1}^{p_{1}} \varphi_{2}^{p_{2}} \cdots \varphi_{k}^{p_{k}} h:\right. \\
\left.h \in \mathscr{H} ; p_{i}, q_{i} \text { are nonnegative integers }\right\} .
\end{aligned}
$$


That $\mathscr{K}^{\prime}$ is closed and $\mathscr{K}^{\prime} \supset \mathscr{H}$ are easy consequences of (2). (The inclusion corresponding to $\mathscr{K}^{\prime} \supset \mathscr{H}$ seems to have been tacitly assumed in [C2].) The inclusion $\mathscr{K}^{\prime \prime} \subset \mathscr{K}^{\prime}$ is justified using the same argument as in [C2, Theorem 3.5]. To prove that $\mathscr{K}^{\prime \prime}$ actually equals $\mathscr{K}^{\prime}$, one chooses $f \in \mathscr{K}^{\prime}$ such that $f$ is orthogonal to $\mathscr{K}^{\prime \prime}$ and shows that $f$ is zero. For this one starts off by considering the inner product $\left\langle\bar{\varphi}_{1}^{q_{1}} \cdots \bar{\varphi}_{k}^{q_{k}} \varphi_{1}^{p_{1}} \cdots \varphi_{k}^{p_{k}} h, f\right\rangle$ instead of $\left\langle\varphi_{1}^{p_{1}} \cdots \varphi_{k}^{p_{k}} h, f\right\rangle$ as in [C2, Theorem 3.5]. The rest of the argument remains essentially the same as there.

Corollary 1. In the notation of Theorem $3, \varphi(N)$ is a minimal normal dilation of $\varphi(S)$ if and only if $\mathscr{K}_{\xi}=L^{2}\left(\lambda_{\xi}\right)$ a.e. $\left[\mu \circ \varphi^{-1}\right]$.

Remark 1. When $N$ is a minimal normal extension of $S$ and $\varphi$ belongs to the algebra $\mathscr{B}(S)^{(k)}$ as defined in [C2], Theorem 3 in effect reduces to [C2, Theorem 3.5]. The assumption about the minimality of $N$ in [C2] is not always essential. It is, however, handy in certain cases such as when $\varphi_{j}$ are rational functions with poles off the joint Taylor spectrum of $S$. (For the particular case mentioned here, interpreting $\varphi(N)$ poses no problem in view of the spectral inclusion property for subnormal tuples [P].) In all of the examples discussed below, the normal extensions $N$ of $S$ turn out to be minimal in view of the Stone-Weierstrass theorem.

\section{Applications}

If $X$ is a compact subset of $\mathbb{C}^{m}$ and $f$ is a continuous function from $X$ into $\mathbb{C}^{m}$, then it is easy to see that $X$ is homeomorphic to $Y=\{(z, f(z)): z \in X\}$, the graph of $X$, under the map $z \rightarrow(z, f(z))$. It follows that $C(X)$ is isometrically isomorphic to $C(Y)$. This observation will be helpful in interpreting the conclusions of some of the theorems quoted below, which are slight reformulations of those stated originally. Observe that if polynomials are uniformly dense in $C(Y)$, then so are they in $C\left(Y^{\prime}\right)$ for any closed subset $Y^{\prime}$ of $Y$. If $\mu$ is a compactly supported regular Borel measure in $\mathbb{C}^{m}$, then $P^{2}(\mu)$ denotes the closure of polynomials in $L^{2}(\mu)$. If $f$ is a sufficiently smooth function, then, writing $z=x+i y, f_{z}$ stands for $\frac{1}{2}(\partial f / \partial x-i \partial f / \partial y)$ and $f_{\bar{z}}$ for $\frac{1}{2}(\partial f / \partial x+i \partial f / \partial y)$. The symbol $\mathbb{D}^{m}$ will denote the closed unit polydisk in $\mathbb{C}^{m}$

If $\mu$ is a compactly supported measure in $\mathbb{C}^{m}$ and $N_{i}\left(S_{i}\right)$ is multiplication by the coordinate function $z_{i}$ on $L^{2}(\mu) \quad\left(P^{2}(\mu)\right)$, then $N=\left(N_{1}, \ldots, N_{m}\right)$ $\left(S=\left(S_{1}, \ldots, S_{m}\right)\right)$ will be referred to as the multiplication tuple on $L^{2}(\mu)$ $\left(P^{2}(\mu)\right)$. Theorem 4 is due to Hörmander and Wermer (see also [W1, W2]), and the rest of the theorems are due to Preskenis (see also [O'FP]). For $z=$ $\left(z_{1}, \ldots, z_{m}\right)$ in $\mathbb{C}^{m},|z|$ stands for $\sqrt{\left|z_{1}\right|^{2}+\cdots+\left|z_{m}\right|^{2}}$.

Theorem 4 [HW]. Let $X$ be a compact subset of $\mathbb{C}^{m}$, and let $\Omega$ be an open neighbourhood of $X$. Also let $R=\left(R_{1}, \ldots, R_{m}\right)$ be a vector-valued function defined on $\Omega$. Assume there exists a positive $k<1$ such that $\left|R(z)-R\left(z^{\prime}\right)\right| \leq$ $k\left|z-z^{\prime}\right|$ if $z, z^{\prime} \in \Omega$. If each $R_{j}$ is an $(m+2)$-times continuously differentiable function, then the polynomials in $z_{1}, \ldots, z_{m}$ and $\bar{z}_{1}+R_{1}, \ldots, \bar{z}_{m}+R_{m}$ are dense in $C(X)$.

Proposition 1. Let $N=\left(N_{1}, \ldots, N_{m}\right)$ be the multiplication tuple on $L^{2}(\mu)$ and $S=\left(S_{1}, \ldots, S_{m}\right)$ be that on $P^{2}(\mu)$. Also let $R_{1}, \ldots, R_{m}$ be analytic 
polynomials such that $R=\left(R_{1}, \ldots, R_{m}\right)$ satisfies the hypotheses of Theorem 4 (with $X=$ support of $\mu$ ). If $\varphi=\left(z_{1}+R_{1}, \ldots, z_{m}+R_{m}\right)$, then $\varphi(N)$ is a minimal normal extension of $\varphi(S) \equiv \varphi(N) / P^{2}(\mu)$ and $\varphi(N)^{*}$ is a minimal normal dilation of $\varphi\left(S^{*}\right)$.

Remark 2. Statements such as those in Proposition 1 are obvious in view of (1) and an appropriate approximation theorem in several complex variables. Hereafter, we will not dwell on such straightforward applications of (1), though it must be mentioned that corresponding to every theorem mentioned below, a similar result as in Proposition 1 is valid. Our main interest lies in the application of Theorem 3 (or Corollary 1) to derive less obvious results. Note that the conclusion of Theorem 4 can be reformulated as follows: The $2 m$-variable analytic polynomials are uniformly dense in $C\{(z, \bar{z}+R(z)): z \in X\}$.

Proposition 2. For $\mu$ supported on $\mathbb{D}^{2 m}$, let $N=\left(N_{1}, \ldots, N_{2 m}\right)$ be the multiplication tuple on $L^{2}(\mu)$ and $S=\left(S_{1}, \ldots, S_{2 m}\right)$ be that on $P^{2}(\mu)$, with $S$ being doubly commuting. Let $\varphi_{j}=z_{j+m}-\left(\bar{z}_{j}+R_{j}\right) \quad(j=1, \ldots, m)$, where $R=\left(R_{1}, \ldots, R_{m}\right)$ satisfies the hypotheses of Theorem 4 with $X=\mathbb{D}^{m}$ and each $R_{j}$ is just a polynomial in $\bar{z}_{j}$. Then $\varphi(N)$ is a minimal normal dilation of $\varphi(S) \equiv P\left(L^{2}(\mu), P^{2}(\mu)\right) \varphi(N) \mid P^{2}(\mu)$.

Proof. Let $\mathscr{H}_{0} \subset P^{2}(\mu)$ be the (dense) set of $2 m$-variable analytic polynomials with complex rational coefficients. By Corollary 1 it suffices to show that $L^{2}\left(\lambda_{\xi}\right)=\mathscr{K}_{\xi}$ a.e. $\left[\mu \circ \varphi^{-1}\right] .\left(\xi=\left(\xi_{1}, \ldots, \xi_{m}\right) \in \varphi(\right.$ support of $\mu) \subset \mathbb{C}^{m}$.) Now $\mathscr{K}_{\xi}=L^{2}\left(\lambda_{\xi}\right)$-closure of $\left\{h / \varphi^{-1}(\xi): h \in \mathscr{L}_{0}\right\}$, and $\lambda_{\xi}$ is supported on

$$
\begin{aligned}
\varphi^{-1}(\xi) & =\left\{z=\left(z_{1}, \ldots, z_{2 m}\right) \in \text { support of } \mu: \varphi_{j}(z)=\xi_{j}\right\} \\
& =\left\{z \in \text { support of } \mu: z_{j+m}=\bar{z}_{j}+R_{j}+\xi_{j}\right\} .
\end{aligned}
$$

Since $R=\left(R_{1}+\xi_{1}, \ldots, R_{m}+\xi_{m}\right)$ satisfies the hypotheses of Theorem 4, it follows that $\left\{h / \varphi^{-1}(\xi): h \in \mathscr{L}_{0}\right\}$ is dense in $C\left(\varphi^{-1}(\xi)\right)$ in the uniform norm and hence dense in $L^{2}\left(\lambda_{\xi}\right)$ for every $\xi$ in $\varphi$ (support of $\mu$ ).

Remark 3. For $X=\mathbb{D}$ the hypotheses of Theorem 4 can be somewhat weakened; specifically, it suffices to assume that $\left|R(z)-R\left(z^{\prime}\right)\right|<\left|z-z^{\prime}\right|$ for $z, z^{\prime} \in \mathbb{D}$ with $z \neq z^{\prime}$ (see [W1]). Letting $R_{1}\left(z_{1}\right)=\bar{z}_{1}^{2} / 2$, it follows in particular that $N_{2}-\left(N_{1}^{*}+N_{1}^{* 2} / 2\right)$ is a minimal normal dilation of $S_{2}-\left(S_{1}^{*}+S_{1}^{* 2} / 2\right)$, where $S=\left(S_{1}, S_{2}\right)$ is the multiplication pair on the Hardy (or Bergman) space of the closed unit bidisk $\mathbb{D}^{2}$ and $N=\left(N_{1}, N_{2}\right)$ is the multiplication pair on $L^{2}(\mu)$, $\mu$ being the product arc-length measure on the distinguished boundary of $\mathbb{D}^{2}$ (or $\mu$ being the product area measure on $\mathbb{D}^{2}$ ).

Theorem $5[\mathrm{P} 1]$. Let $f \in C(\mathbb{D})$ be such that

(i) $f$ is differentiable on the interior of $\mathbb{D}$,

(ii) $\operatorname{Re} f_{\bar{z}} \geq\left|f_{z}\right|$ on the interior of $\mathbb{D}$, and

(iii) $f^{-1}(f(a))$ is countable for each $a$ in $\mathbb{D}$.

Then the two-variable analytic polynomials are dense in $C(\{(z, f(z): z \in \mathbb{D}\})$.

Proposition 3. For $\mu$ supported on $\mathbb{D}^{2}$, let $N=\left(N_{1}, N_{2}\right)$ be the multiplication pair on $L^{2}(\mu)$ and let $S=\left(S_{1}, S_{2}\right)$ be that on $P^{2}(\mu)$, with $S$ being doubly commuting. If $f(z)$ is a polynomial in $\bar{z}$ such that $\operatorname{Re} f_{\bar{z}} \geq 0$ on the interior 
of $\mathbb{D}$ and $\varphi\left(z_{1}, z_{2}\right)=z_{2}-f\left(z_{1}\right)$, then $\varphi(N)$ is a minimal normal dilation of $\varphi(S)$.

Proof. Let $\mathscr{L}_{0} \subset P^{2}(\mu)$ be the (dense) set of 2-variable analytic polynomials with complex rational coefficients. Note that, for $\xi \in \varphi$ (support of $\mu$ ), $\varphi^{-1}(\xi)=\left\{\left(z_{1}, z_{2}\right) \in\right.$ support of $\left.\mu: z_{2}=f\left(z_{1}\right)+\xi\right\}$. Clearly, $g(z)=f(z)+\xi$ is a polynomial in $\bar{z}$ satisfying the hypotheses of Theorem 5 . Thus $\left\{h / \varphi^{-1}(\xi): h\right.$ $\left.\in \mathscr{H}_{0}\right\}$ is dense in $C\left(\varphi^{-1}(\xi)\right)$ and Corollary 1 can be applied.

Remark 4. It is interesting to observe that the example mentioned in Remark 3 is also a consequence of Proposition 3 if one chooses $f(z)=\bar{z}+\bar{z}^{2} / 2$. As another example, consider $\varphi\left(z_{1}, z_{2}\right)=z_{2}-\exp \left(\bar{z}_{1}\right)$. Here $f(z)=\exp (\bar{z})$ is not a polynomial but is easily seen to satisfy the hypotheses of Theorem 5 , so the conclusion of Proposition 3 still holds if $S$ and $N$ are as in Remark 3.

Theorem 6 [P2]. Let $f=\bar{z}^{k} \psi\left(|z|^{2 k}\right)$, where $\psi$ is continuous in [0,1] and differentiable in $(0,1), k$ is a positive integer, and $\left|f_{\bar{z}}\right|>\left|f_{z}\right|$ on (Interior of $\mathbb{D})-\{0\}$. Then the 2-variable analytic polynomials are uniformly dense in $C(\{z, f(z)): z \in \mathbb{D}\})$.

An operator $S$ is called quasi-normal if $S$ and $S^{*} S$ commute.

Proposition 4. For $\mu$ support on $\mathbb{D}^{2}$, let $N=\left(N_{1}, N_{2}\right)$ be the multiplication pair on $L^{2}(\mu)$ and $S=\left(S_{1}, S_{2}\right)$ be that on $P^{2}(\mu)$, with $S$ being doubly commuting and $S_{1}$ being quasi-normal. If $f, \psi$, and $k$ are as in Theorem 6 with $\psi$ being a polynomial, and $\varphi\left(z_{1}, z_{2}\right)=z_{2}-f\left(z_{1}\right)$, then $\varphi(N)$ is a minimal normal dilation of $\varphi(S)$.

Proof. Let $f\left(z_{1}\right)=\bar{z}_{1}^{k}\left(a_{0}+a_{1}\left|z_{1}\right|^{2 p_{1} k}+\cdots+a_{n}\left|z_{1}\right|^{2 p_{n} k}\right)$, where $p_{i}$ are positive integers. Thus

$$
\varphi(N)=N_{2}-N_{1}^{* k}\left(a_{0}+a_{1} N_{1}^{*_{1}^{p} k} N_{1}^{p_{1} k}+\cdots+a_{n} N^{*_{n}^{p} k} N_{1}^{p_{n} k}\right) .
$$

The assumption of quasi normality of $S_{1}$ guarantees that

$$
\left(S_{i}^{*\left(p_{i}+1\right) k} S_{1}^{p_{i} k}\right)\left(S_{1}^{\left.* p_{j}+1\right) k} S_{1}^{p_{j} k}\right)=S_{1}^{*^{\left(p_{i}+p_{j}+2\right) k}} S_{1}^{\left(p_{j}+p_{j}\right) k} .
$$

(This requires an elementary verification.) Combining this observation with the assumption of "doubly commuting", it follows that $\varphi(N)$ is a normal dilation of $\varphi(S)$. It is clear that the function $f$ in Theorem 6 may be replaced by $f_{1}=f+\xi$ without affecting the conclusion there. (Any polynomial in $z$ and $f_{1}-\xi$ is a polynomial in $z$ and $f_{1}$.) The reader can now furnish the rest of the argument.

Remark 5. If $f$ is as in the proof of Proposition 4, $a_{0}=0, a_{i}>0$ for all $i$ (or $a_{i}<0$ for all $i$ ), and $p_{1}<p_{2}<\cdots<p_{n}$, then it is easy to check that $f$ satisfies the hypotheses of Theorem 6 . Since the multiplication pair $S=\left(S_{1}, S_{2}\right)$ on the Hardy space of the bidisk is doubly commuting with $S_{1}$ (and $S_{2}$ ) being quasinormal, it is interesting to check Proposition 4 for $S$. Here each $S_{i}$ is actually an isometry and the corresponding $N_{i}$ a unitary; thus the relevant conclusion is that $N_{2}-a N_{1}^{* k}$ is a minimal normal dilation of $S_{2}-a S_{1}^{* k}$ for any nonzero real $a$ and positive integer $k$. The conclusion of Proposition 4 also holds if $S$, for example, is the multiplication pair on the tensor product of the Hardy space of $\mathbb{D}$ with the Bergman space of $\mathbb{D}$ (or the Hardy or Bergman space of the annulus $\{z \in \mathbb{C}: r \leq|z| \leq 1\} \quad(r>0))$. 
In order not to obscure the main ideas, we have avoided introducing too many generalities in the statements of propositions. In Proposition 2 one could let $\varphi_{j}=z_{j+m}-\left(\bar{z}_{j}+R_{j}\right)$, where $R_{j}$ is a polynomial in $\bar{z}_{i_{j}},\left\{i_{1}, \ldots, i_{m}\right\}$ being a permutation of $\{1, \ldots, m\}$; also, the subtuples $\left(S_{1}, \ldots, S_{m}\right)$ and $\left(S_{m+1}, \ldots, S_{2 m}\right)$ are not required to be doubly commuting there. The choice of functions $\varphi$ can be generalized to involve coanalytic functions other than polynomials in $\bar{z}_{j}$. Also, the frequent assumption that $S_{i}$ are contractions can be relaxed. There is one strengthening of the results, however, which demands more attention and is

Proposition 5. In the notation of Proposition 2 and with $m=1$ (or in the notation of Proposition 3 or 4$), p(\varphi(N))$ is a minimal normal dilation of $p(\varphi(S))$, where $p$ is any one-variable polynomial.

Proof. Let $\xi \rightarrow \lambda_{\xi}$ be the disintegration of $\mu$ with respect to $p \circ \varphi$. For $\xi \in$ $(p \circ \varphi)$ (support of $\mu),(p \circ \varphi)^{-1}(\xi)=\left\{\left(z_{1}, z_{2}\right) \in\right.$ support of $\mu: p\left(\varphi\left(z_{1}, z_{2}\right)\right)=$ $\xi\}=\bigcup_{i=1}^{n} F_{i}$, where $F_{i}=\left\{\left(z_{1}, z_{2}\right) \in\right.$ support of $\left.\mu: \varphi\left(z_{1}, z_{2}\right)=\eta_{i}\right\}, \eta_{1}, \ldots$, $\eta_{n}$ being $n$ distinct roots of $p-\xi$. Since our hypotheses guarantee that 2variable analytic polynomials are uniformly dense in $C\left(F_{i}\right)$, it follows by a well-known theorem from the theory of function algebras (see [L, Chapter 3, Theorem 8]) that these are uniformly dense in $C\left(\bigcup_{i=1}^{n} F_{i}\right)$ as well. The rest is obvious.

The above discussion indicates that the methods introduced in [C2] need more investigation and have interesting operator-theoretic implications besides the ones for the minimal normal extension problem as considered in $[\mathrm{C} 1, \mathrm{C} 2$, D].

\section{REFERENCES}

[B] N. Bourbaki, Ėléments des mathématique, Livre VI, Intégration, Chapitre 6, Intégration Vectorielle, Hermann, Paris, 1959.

[C1] J. B. Conway, The minimal normal extension of a function of a subnormal operator, Analysis at Urbana II, Proc. Special year in Modern Analysis at Univ. of Illinois, 1986-87, Cambridge Univ. Press, Cambridge, 1989, pp. 128-140.

[C2] _ Towards a functional calculus for subnormal tuples: the minimal normal extension, Trans. Amer. Math. Soc. 326 (1991), 543-567.

[D] J. Dudziak, The minimal normal extension problem for subnormal operators, J. Funct. Anal. 65 (1986), 314-338.

[HW] L. Hörmander and J. Wermer, Uniform approximation on compact sets in $C^{n}$, Math. Scand. 23 (1968), 5-21.

[I] T. Ito, On the commutative family of subnormal operators, J. Fac. Sci. Hokkaido Univ. 14 (1958), 1-15.

[L] G. M. Leibowitz, Lectures on complex function algebras, Scott, Foresman \& Co., Glenview, IL, 1970.

[O'FP] A. G. O'Farrell and K. J. Preskenis, Approximation by polynomials in two complex variables, Math. Ann. 246 (1980), 225-232.

[P1] K. J. Preskenis, Another view of the Weierstrass theorem, Proc. Amer. Math. Soc. 54 (1976), 109-113.

[P2] - Approximation by polynomials in $z$ and another function, Proc. Amer. Math. Soc. 68 (1978), 69-74. 
[P] M. Putinar, Spectral inclusion for subnormal n-tuples, Proc. Amer. Math. Soc. 90 (1984), 405-406.

[W1] J. Wermer, Approximation on a disc, Math. Ann. 155 (1964), 331-333.

[W2] _ Banach algebras and several complex variables, Springer-Verlag, New York, 1976.

Department of Mathematics, University of Poona, Pune 411 007, India 